Acta Crystallographica Section E

Structure Reports

Online

ISSN 1600-5368

\section{Extensive hydrogen-bonding network and an unusual cation conformation in [tris(hydroxymethyl)methyl]ammonium tetraoxidorhenate(VII)}

\author{
Małgorzata Hołyńska* and Tadeusz Lis \\ Faculty of Chemistry, University of Wrocław, 14 Joliot-Curie St, 50-383 Wrocław, \\ Poland \\ Correspondence e-mail: holynska@wcheto.chem.uni.wroc.pl
}

Received 8 July 2009; accepted 21 July 2009

Key indicators: single-crystal X-ray study; $T=110 \mathrm{~K}$; mean $\sigma(\mathrm{C}-\mathrm{C})=0.005 \AA$. $R$ factor $=0.020 ; w R$ factor $=0.034 ;$ data-to-parameter ratio $=24.0$.

The title compound, $\left(\mathrm{C}_{4} \mathrm{H}_{12} \mathrm{NO}_{3}\right)\left[\mathrm{ReO}_{4}\right]$, contains two cations and two anions in the asymmetric unit, related by a noncrystallographic centre of symmetry. The crystal structure is stabilized by an extensive hydrogen-bonding network with the formation of puckered layers perpendicular to [001]. In the tris(hydroxymethyl)ammonium cations, intramolecular $\mathrm{O}-$ $\mathrm{H}$... O hydrogen bonds are present with the formation of an $S_{1}^{1}(6)$ graph-set motif. The crystal structure is further consolidated by $\mathrm{N}-\mathrm{H} \cdots \mathrm{O}$ hydrogen bonds.

\section{Related literature}

For related structures, see: Castellari \& Ottani (1997); Eilerman \& Rudman (1980); Hołyńska \& Lis (2004, 2008); Lock \& Turner (1975); Marsh et al. (1998); Rudman et al. (1979, 1983); Shakked et al. (1980); Tusvik et al. (1999). For the dielectric properties of rhenates(VII) with organic ammonium cations, see: Czarnecki \& Małuszyńska (2000). For graph-set notation, see: Etter et al. (1990). For the synthesis of rhenic(VII) acid, see: Johnson et al. (1967).

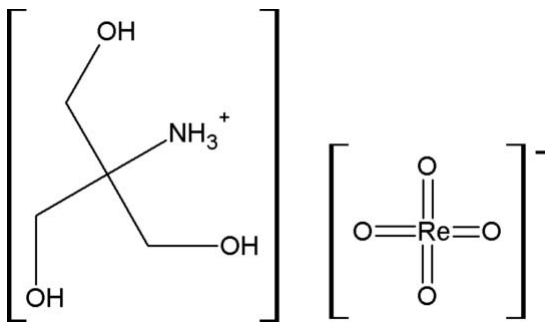

\section{Experimental}

Crystal data

$\left(\mathrm{C}_{4} \mathrm{H}_{12} \mathrm{NO}_{3}\right)\left[\mathrm{ReO}_{4}\right]$

$M_{r}=372.35$

Orthorhombic, $P c a 2_{1}$

$a=21.450(5) \AA$

$b=6.867$ (2) $\AA$

$c=12.219(4) \AA$

Data collection

Oxford Diffraction KM-4-CCD diffractometer

Absorption correction: analytical

(CrysAlis RED; Oxford

Diffraction, 2006)

$T_{\min }=0.104, T_{\max }=0.268$

\section{Refinement}

$R\left[F^{2}>2 \sigma\left(F^{2}\right)\right]=0.020$

$w R\left(F^{2}\right)=0.034$

$S=1.02$

5888 reflections

245 parameters

$V=1799.8(9) \AA^{3}$

$Z=8$

Mo $K \alpha$ radiation

$\mu=13.51 \mathrm{~mm}^{-1}$

$T=110 \mathrm{~K}$

$0.21 \times 0.16 \times 0.14 \mathrm{~mm}$

24604 measured reflections 5888 independent reflections 5084 reflections with $I>2 \sigma(I)$ $R_{\text {int }}=0.029$

Table 1

Selected geometric parameters $\left(\AA{ }^{\circ}\right)$.

\begin{tabular}{llll}
\hline $\mathrm{Re} 1-\mathrm{O} 11$ & $1.736(2)$ & $\mathrm{Re} 2-\mathrm{O} 12$ & $1.728(4)$ \\
$\mathrm{Re} 1-\mathrm{O} 21$ & $1.728(2)$ & $\mathrm{Re} 2-\mathrm{O} 22$ & $1.730(3)$ \\
$\mathrm{Re} 1-\mathrm{O} 31$ & $1.727(2)$ & $\mathrm{Re} 2-\mathrm{O} 32$ & $1.736(3)$ \\
$\mathrm{Re} 1-\mathrm{O} 41$ & $1.702(5)$ & $\mathrm{Re} 2-\mathrm{O} 42$ & $1.726(2)$ \\
& & & \\
$\mathrm{O} 41-\mathrm{Re} 1-\mathrm{O} 31$ & $109.6(2)$ & $\mathrm{O} 42-\mathrm{Re} 2-\mathrm{O} 12$ & $108.4(2)$ \\
$\mathrm{O} 41-\mathrm{Re} 1-\mathrm{O} 21$ & $108.7(2)$ & $\mathrm{O} 42-\mathrm{Re} 2-\mathrm{O} 22$ & $109.4(2)$ \\
$\mathrm{O} 31-\mathrm{Re} 1-\mathrm{O} 21$ & $108.7(2)$ & $\mathrm{O} 12-\mathrm{Re} 2-\mathrm{O} 22$ & $109.6(2)$ \\
$\mathrm{O} 41-\mathrm{Re} 1-\mathrm{O} 11$ & $110.6(2)$ & $\mathrm{O} 42-\mathrm{Re} 2-\mathrm{O} 32$ & $109.3(2)$ \\
$\mathrm{O} 31-\mathrm{Re} 1-\mathrm{O} 11$ & $110.0(2)$ & $\mathrm{O} 12-\mathrm{Re} 2-\mathrm{O} 32$ & $110.8(2)$ \\
$\mathrm{O} 21-\mathrm{Re} 1-\mathrm{O} 11$ & $109.2(2)$ & $\mathrm{O} 22-\mathrm{Re} 2-\mathrm{O} 32$ & $109.3(2)$ \\
\hline
\end{tabular}

Table 2

Hydrogen-bond geometry $\left(\AA,^{\circ}\right)$.

\begin{tabular}{|c|c|c|c|c|}
\hline$D-\mathrm{H} \cdots A$ & $D-\mathrm{H}$ & $\mathrm{H} \cdots A$ & $D \cdots A$ & $D-\mathrm{H} \cdots A$ \\
\hline $\mathrm{N} 2-\mathrm{H} 2 A \cdots \mathrm{O} 21$ & 0.91 & 2.03 & $2.858(4)$ & 150 \\
\hline $\mathrm{N} 2-\mathrm{H} 2 B \cdots \mathrm{O} 111^{\mathrm{i}}$ & 0.91 & 1.88 & $2.788(4)$ & 173 \\
\hline $\mathrm{N} 2-\mathrm{H} 2 C \cdots \mathrm{O} 31^{\mathrm{ii}}$ & 0.91 & 1.98 & $2.879(4)$ & 169 \\
\hline $\mathrm{O} 112-\mathrm{H} 112 \cdots \mathrm{O} 212$ & 0.84 & 2.12 & $2.773(4)$ & 134 \\
\hline $\mathrm{O} 112-\mathrm{H} 112 \cdots \mathrm{O} 41$ & 0.84 & 2.49 & $2.942(4)$ & 115 \\
\hline $\mathrm{O} 212-\mathrm{H} 212 \cdots \mathrm{O} 32^{\mathrm{ii}}$ & 0.84 & 1.89 & $2.721(4)$ & 168 \\
\hline $\mathrm{O} 312-\mathrm{H} 312 \cdots \mathrm{O} 112^{\mathrm{ii}}$ & 0.84 & 1.92 & $2.704(4)$ & 156 \\
\hline $\mathrm{N} 1-\mathrm{H} 1 A \cdots \mathrm{O} 312^{\mathrm{iii}}$ & 0.91 & 1.83 & $2.738(4)$ & 176 \\
\hline $\mathrm{N} 1-\mathrm{H} 1 B \cdots \mathrm{O} 42^{\mathrm{ii}}$ & 0.91 & 2.05 & $2.872(4)$ & 150 \\
\hline $\mathrm{N} 1-\mathrm{H} 1 C \cdots \mathrm{O} 22$ & 0.91 & 1.98 & $2.862(4)$ & 164 \\
\hline $\mathrm{O} 111-\mathrm{H} 111 \cdots \mathrm{O} 211^{\mathrm{iv}}$ & 0.84 & 1.89 & $2.681(3)$ & 157 \\
\hline $\mathrm{O} 211-\mathrm{H} 211 \cdots \mathrm{O} 311$ & 0.84 & 2.13 & $2.774(4)$ & 134 \\
\hline $\mathrm{O} 211-\mathrm{H} 211 \cdots \mathrm{O} 12^{\mathrm{ii}}$ & 0.84 & 2.54 & $2.960(4)$ & 112 \\
\hline O311-H311‥ & 0.84 & 1.88 & $2.714(4)$ & 170 \\
\hline
\end{tabular}

Data collection: CrysAlis CCD (Oxford Diffraction, 2006); cell refinement: CrysAlis RED (Oxford Diffraction, 2006); data reduction: CrysAlis RED; program(s) used to solve structure: SHELXS97 (Sheldrick, 2008); program(s) used to refine structure: SHELXL97 (Sheldrick, 2008); molecular graphics: DIAMOND (Brandenburg \& Putz, 2005) and SHELXTL-NT (Sheldrick, 2008); software used to prepare material for publication: SHELXL97. 
Financial support from the Ministry of Science and Higher Education (project No. N204 130 32/3318) is acknowledged.

Supplementary data and figures for this paper are available from the IUCr electronic archives (Reference: EZ2176).

\section{References}

Brandenburg, K. \& Putz, H. (2005). DIAMOND. Crystal Impact GbR, Bonn, Germany.

Castellari, C. \& Ottani, S. (1997). Acta Cryst. C53, 482-486.

Czarnecki, P. \& Małuszyńska, H. (2000). J. Phys. Condens. Matter, 12, 48814892.

Eilerman, D. \& Rudman, R. (1980). J. Chem. Phys. 72, 5656-5666.

Etter, M. C., MacDonald, J. C. \& Bernstein, J. (1990). Acta Cryst. B46, 256-262.
Hołyńska, M. \& Lis, T. (2004). Acta Cryst. C60, m648-m650.

Hołyńska, M. \& Lis, T. (2008). Acta Cryst. C64, i18-i20.

Johnson, N. P., Lock, C. J. L. \& Wilkinson, G. (1967). Inorg. Synth. 9, 145-148.

Lock, C. J. L. \& Turner, G. (1975). Acta Cryst. B31, 1764-1765.

Marsh, R. E., Schomaker, V. \& Herbstein, F. H. (1998). Acta Cryst. B54, $921-$ 924.

Oxford Diffraction (2006). CrysAlis RED and CrysAlis CCD. Oxford Diffraction Ltd, Abingdon, England.

Rudman, R., Lippman, R. \& Eilerman, D. (1979). American Crystallographic Association Abstracts, Winter Meeting, Honolulu, HI, Vol. 6, No. 2, p. 73.

Rudman, R., Lippman, R., Sake Gowda, D. S. \& Eilerman, D. (1983). Acta Cryst. C39, 1267-1271.

Shakked, Z., Viswamitra, M. A. \& Kennard, O. (1980). Biochemistry, 19, $2567-$ 2571.

Sheldrick, G. M. (2008). Acta Cryst. A64, 112-122.

Tusvik, P. H., Mostad, A., Dalhus, B. \& Rosenqvist, E. (1999). Acta Cryst. C55, $1113-1115$. 


\section{supporting information}

Acta Cryst. (2009). E65, m998-m999［doi:10.1107/S1600536809028797]

\section{Extensive hydrogen-bonding network and an unusual cation conformation in [tris(hydroxymethyl)methyl]ammonium tetraoxidorhenate(VII)}

\section{Malgorzata Holyńska and Tadeusz Lis}

\section{S1. Comment}

The title compound was obtained as starting material for other syntheses (e.g. reaction with acethyl chloride - Hołyńska $\&$ Lis, 2008). It was chosen as the tris(hydroxymethyl)methylammonium cation gives rise to an extensive hydrogen bonding network, which allows for selective crystallization of products containing Re, reducing the risk of cocrystallization of impurities and crystal structure disorder. Moreover, rhenates(VII) with organic ammonium cations crystallizing in non-centrosymmetric space groups are promising materials with respect to their dielectric properties. For example, the previously discovered ferroelectric with a Curie temperature above room temperature is pyridinium rhenate(VII) (Czarnecki \& Małuszyńska, 2000).

The title compound (1) is a product of the reaction of rhenic(VII) acid with tris(hydroxymethyl)methylamine (TRIS) in aqueous solution, comprising discrete tris(hydroxymethyl)methylammonium cations (protonated TRIS here denoted as TRISH $^{+}$) and rhenate(VII) anions (Fig. 1, Scheme 1).

There are two symmetry-independent rhenate(VII) anions (containing atoms $\operatorname{Re} 1$ and Re2, respectively) with the expected (see e.g. Hołyńska \& Lis, 2004 for example of rhenate(VII) anions in low symmetry environment) slightly distorted tetrahedral geometry. The $\mathrm{Re}-\mathrm{O}$ bond lengths are listed in Table 1 . Their values are consistent with those for other rhenates(VII), e.g. 1.723 (4) $\AA$ for potassium rhenate(VII) reported by Lock \& Turner (1975). These bond lengths are not much affected by the presence of hydrogen bonds, as all rhenate(VII) $\mathrm{O}$ atoms participate in these interactions as acceptors (Table 2).

It is interesting to note that both symmetry-independent $\mathrm{TRISH}^{+}$cations are of unusual conformation. Usually the cation symmetry is close to $\mathrm{C}_{3}$ (e.g. Rudman et al., 1983) or even exactly threefold (as in [TRISH]Cl appearing in a preliminary report by Rudman et al., 1979) with no intramolecular hydrogen bonds. In (1) both cations exhibit the presence of such intramolecular hydrogen bond (Table 2) with the formation of a $\mathrm{S}_{1}{ }^{1}(6)$ graph-set motif (Etter et al., 1990). The relevant $\mathrm{N}$ $-\mathrm{C}-\mathrm{C}-\mathrm{O}$ torsion angles are given in Table 1 . Bond lengths characterizing the cations, among them the $\mathrm{C}-\mathrm{N}$ bond length (which is longer than in the TRIS molecule - 1.477 (3) $\AA$ as reported for the neutral TRIS molecule by Eilerman \& Rudman, 1980) are in accordance with the values reported for other structures (e.g. Castellari \& Ottani, 1997). TRIS is a constituent of buffers used in biochemical studies in the $\mathrm{pH}$ range of 7-9 (Castellari \& Ottani, 1997). Upon protonation it forms salts with biologically relevant anions (e.g. tris(hydroxymethyl)methylammonium deoxycholate reported by Tusvik et al., 1999), also a report on its interaction with nucleotides in the crystalline state is available (Shakked et al., 1980).

All cation ammonium and hydroxyl groups are donors in $\mathrm{N}-\mathrm{H} \cdots \mathrm{O}$ or $\mathrm{O}-\mathrm{H} \cdots \mathrm{O}$ hydrogen bonds, both to other TRISH ${ }^{+}$ cations or to rhenate(VII) anions. The shortest Re $\cdots \operatorname{Re}$ distance is 4.210 (2) $\AA$. Thus, puckered hydrogen-bonded layers perpendicular to [001] are formed (Fig. 2). The hydrogen bonding scheme stabilizing an individual layer is illustrated in Fig. 3. 


\section{S2. Experimental}

The title compound was obtained in the reaction of $0.19 \mathrm{~g}$ of tris(hydroxymethyl)methylamine (TRIS) with an excess of rhenic(VII) acid in aqueous solution, with slow evaporation leading to colourless crystals. The reaction was carried out in a quartz beaker. Rhenic(VII) acid was obtained according to the literature procedure (Johnson et al., 1967) in reaction of $0.3 \mathrm{~g}$ of metallic Re with an excess of a $30 \%$ aqueous hydrogen peroxide solution.

\section{S3. Refinement}

The structure was solved by direct methods in the space group P1, and the present solution was obtained by switching to a higher symmetry. It was possible to end up in a false minimum in the $\mathrm{Pca} 2_{1}$ space group (e.g. with the following approximate coordinates for the Re atoms: 0.57, 0.024, 0.97 for Re1; 0.69, 0.51, 1.01 for Re2; see Marsh et al., 1998, for a review of some pitfalls connected with the Pca $2_{1}$ space group). All $\mathrm{H}$ atoms were generated geometrically and refined with $U_{\text {eq }}=\mathrm{n} U_{\text {eq }}$ (parent atom), where $\mathrm{n}=1.5$ for hydroxyl $\mathrm{H}$ atoms, and $\mathrm{n}=1.2$ for the remaining $\mathrm{H}$ atoms. During the refinement, extinction was also taken into account. Furthermore, it was found that the structure is a racemic twin (with a refined BASF parameter value of 0.375 (6)). On the final difference Fourier map the highest peak of $2.00 \mathrm{e} / \AA^{3}$ was found at $0.62 \AA$ from atom Re2. The crystal structure contains a pseudosymmetry centre at approximately $(0.37,0.73,0.38)$.

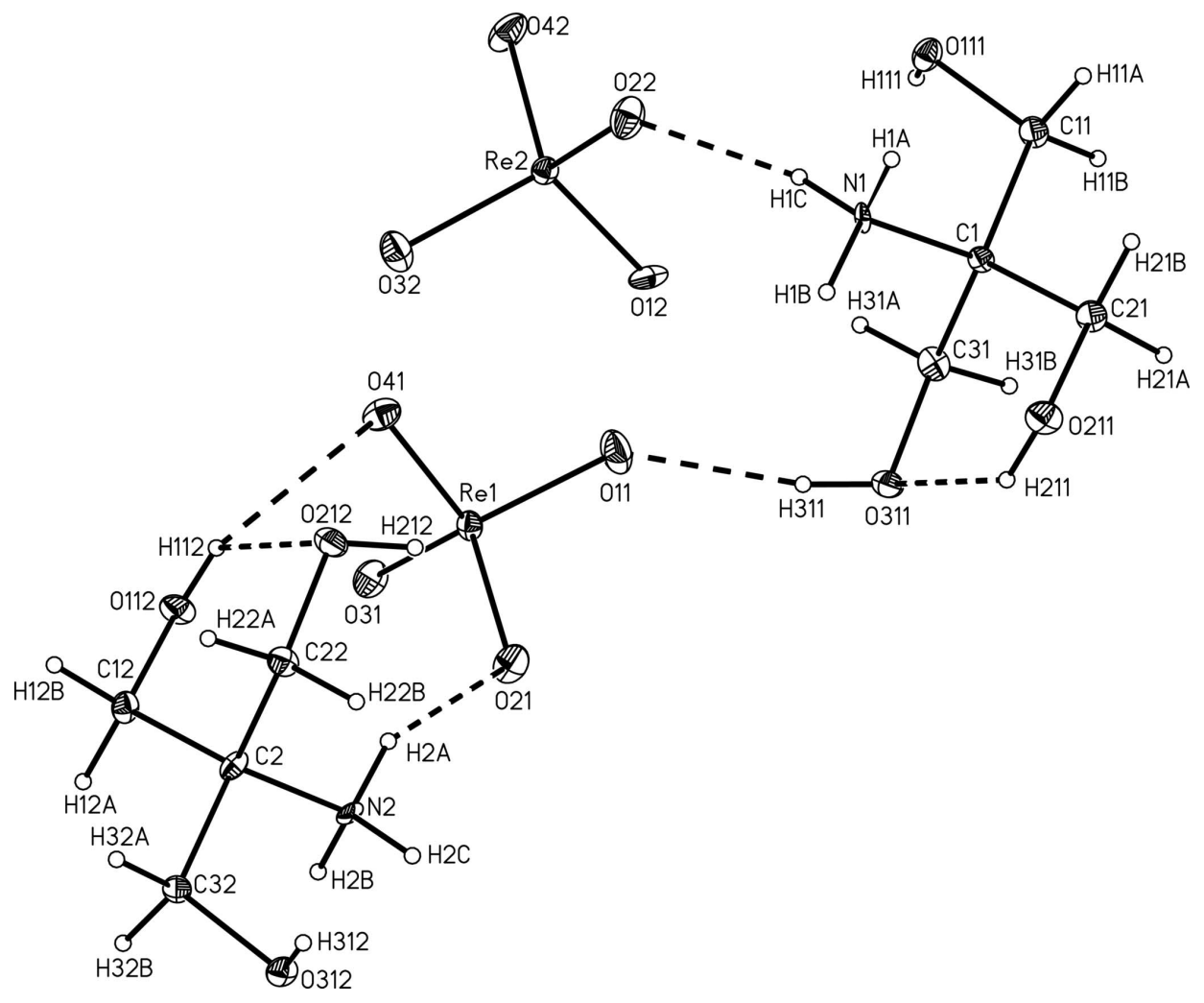

\section{Figure 1}

The symmetry-independent part of (1). Thermal ellipsoids are drawn at 30\% probability level. Hydrogen bonds are denoted with dashed lines. 


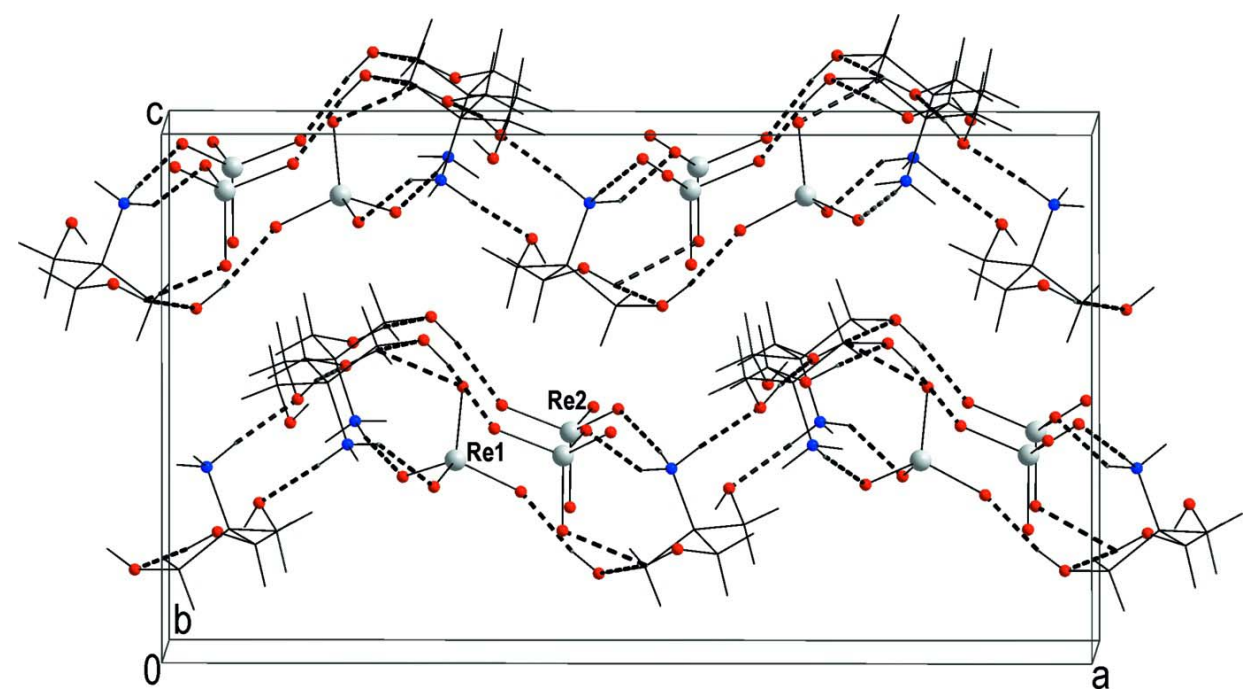

Figure 2

Puckered hydrogen-bonded layers perpendicular to [001]. Hydrogen bonds are denoted with dashed lines.

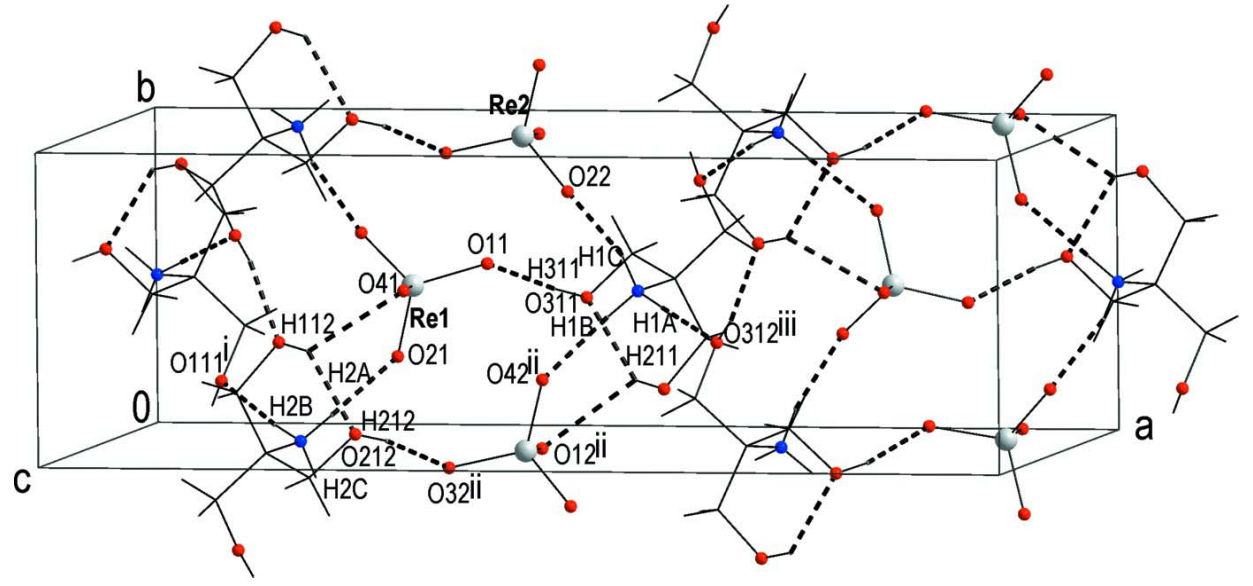

\section{Figure 3}

One of the layers with hydrogen bonding scheme. Hydrogen bonds are denoted with dashed lines. Symmetry codes as in Table 2.

\section{[tris(hydroxymethyl)methyl]ammonium tetraoxidorhenate(VII)}

\section{Crystal data}

$\left(\mathrm{C}_{4} \mathrm{H}_{12} \mathrm{NO}_{3}\right)\left[\mathrm{ReO}_{4}\right]$

$M_{r}=372.35$

Orthorhombic, $P c a 2_{1}$

Hall symbol: P 2c -2ac

$a=21.450(5) \AA$

$b=6.867(2) \AA$

$c=12.219(4) \AA$

$V=1799.8(9) \AA^{3}$

$Z=8$
$F(000)=1392$

$D_{\mathrm{x}}=2.748 \mathrm{Mg} \mathrm{m}^{-3}$

Mo $K \alpha$ radiation, $\lambda=0.71073 \AA$

Cell parameters from 20472 reflections

$\theta=4.2-35.0^{\circ}$

$\mu=13.51 \mathrm{~mm}^{-1}$

$T=110 \mathrm{~K}$

Needle, colourless

$0.21 \times 0.16 \times 0.14 \mathrm{~mm}$ 


\section{Data collection}

Oxford Diffraction KM-4-CCD diffractometer

Radiation source: fine-focus sealed tube Graphite monochromator $\omega$ scans

Absorption correction: analytical

(CrysAlis RED; Oxford Diffraction, 2006)

$T_{\min }=0.104, T_{\max }=0.268$

\section{Refinement}

Refinement on $F^{2}$

Least-squares matrix: full

$R\left[F^{2}>2 \sigma\left(F^{2}\right)\right]=0.020$

$w R\left(F^{2}\right)=0.034$

$S=1.02$

5888 reflections

245 parameters

1 restraint

Primary atom site location: structure-invariant direct methods

Secondary atom site location: difference Fourier map
24604 measured reflections

5888 independent reflections

5084 reflections with $I>2 \sigma(I)$

$R_{\text {int }}=0.029$

$\theta_{\max }=35.0^{\circ}, \theta_{\min }=4.2^{\circ}$

$h=-33 \rightarrow 28$

$k=-9 \rightarrow 11$

$l=-14 \rightarrow 19$

Hydrogen site location: inferred from neighbouring sites

$\mathrm{H}$-atom parameters constrained

$w=1 /\left[\sigma^{2}\left(F_{\mathrm{o}}{ }^{2}\right)+(0.0119 P)^{2}\right]$

where $P=\left(F_{\mathrm{o}}^{2}+2 F_{\mathrm{c}}{ }^{2}\right) / 3$

$(\Delta / \sigma)_{\max }=0.002$

$\Delta \rho_{\max }=2.00 \mathrm{e}^{-3}$

$\Delta \rho_{\min }=-1.27 \mathrm{e}^{-3}$

Extinction correction: SHELXL97 (Sheldrick, 2008), $\mathrm{Fc}^{*}=\mathrm{kFc}\left[1+0.001 \mathrm{xFc}^{2} \lambda^{3} / \sin (2 \theta)\right]^{-1 / 4}$

Extinction coefficient: 0.00101 (3)

Fractional atomic coordinates and isotropic or equivalent isotropic displacement parameters $\left(\AA^{2}\right)$

\begin{tabular}{lllll}
\hline & $x$ & $y$ & $z$ & $U_{\text {iso }} / U_{\text {eq }}$ \\
\hline Re1 & $0.311748(6)$ & $0.494021(19)$ & $0.362409(13)$ & $0.01257(4)$ \\
O11 & $0.38162(12)$ & $0.5562(4)$ & $0.2998(2)$ & $0.0266(6)$ \\
O21 & $0.29045(12)$ & $0.2623(3)$ & $0.3220(2)$ & $0.0217(6)$ \\
O31 & $0.25389(12)$ & $0.6547(3)$ & $0.3237(2)$ & $0.0204(6)$ \\
O41 & $0.31950(13)$ & $0.4971(3)$ & $0.5010(4)$ & $0.0192(7)$ \\
Re2 & $0.431704(6)$ & $0.975939(17)$ & $0.393938(10)$ & $0.01252(4)$ \\
O12 & $0.43101(13)$ & $0.9629(4)$ & $0.2527(4)$ & $0.0201(7)$ \\
O22 & $0.48425(12)$ & $0.8084(4)$ & $0.4456(2)$ & $0.0239(6)$ \\
O32 & $0.35819(12)$ & $0.9281(4)$ & $0.4468(2)$ & $0.0236(6)$ \\
O42 & $0.45440(14)$ & $1.2076(3)$ & $0.4316(2)$ & $0.0256(6)$ \\
N1 & $0.54415(13)$ & $0.4794(3)$ & $0.3475(3)$ & $0.0087(7)$ \\
H1A & 0.5752 & 0.4330 & 0.3909 & $0.010^{*}$ \\
H1B & 0.5116 & 0.3943 & 0.3478 & $0.010^{*}$ \\
H1C & 0.5312 & 0.5969 & 0.3733 & $0.010^{*}$ \\
C1 & $0.56824(16)$ & $0.5031(4)$ & $0.2313(5)$ & $0.0108(9)$ \\
C11 & $0.62070(17)$ & $0.6555(5)$ & $0.2341(3)$ & $0.0158(8)$ \\
H11A & 0.6583 & 0.5969 & 0.2674 & $0.019^{*}$ \\
H11B & 0.6313 & 0.6942 & 0.1582 & $0.019^{*}$ \\
O111 & $0.60331(12)$ & $0.8244(3)$ & $0.2949(2)$ & $0.0161(5)$ \\
H111 & 0.5882 & 0.9079 & 0.2523 & $0.024^{*}$ \\
C21 & $0.59498(17)$ & $0.3079(5)$ & $0.1935(3)$ & $0.0161(8)$ \\
H21A & 0.6055 & 0.3169 & 0.1148 & $0.019^{*}$ \\
H21B & 0.6341 & 0.2820 & 0.2340 & $0.019^{*}$ \\
O211 & $0.55315(12)$ & $0.1482(3)$ & $0.2097(2)$ & $0.0190(6)$
\end{tabular}




\begin{tabular}{lllll}
$\mathrm{H} 211$ & 0.5192 & 0.1715 & 0.1780 & $0.029^{*}$ \\
$\mathrm{C} 31$ & $0.51492(18)$ & $0.5698(6)$ & $0.1573(3)$ & $0.0171(8)$ \\
$\mathrm{H} 31 \mathrm{~A}$ & 0.4977 & 0.6936 & 0.1858 & $0.020^{*}$ \\
$\mathrm{H} 31 \mathrm{~B}$ & 0.5313 & 0.5947 & 0.0829 & $0.020^{*}$ \\
$\mathrm{O} 311$ & $0.46638(12)$ & $0.4291(4)$ & $0.1512(2)$ & $0.0194(6)$ \\
$\mathrm{H} 311$ & 0.4369 & 0.4628 & 0.1922 & $0.029^{*}$ \\
$\mathrm{~N} 2$ & $0.19985(14)$ & $0.0038(3)$ & $0.4133(3)$ & $0.0106(7)$ \\
$\mathrm{H} 2 \mathrm{~A}$ & 0.2325 & 0.0881 & 0.4100 & $0.013^{*}$ \\
$\mathrm{H} 2 \mathrm{~B}$ & 0.1679 & 0.0501 & 0.3718 & $0.013^{*}$ \\
$\mathrm{H} 2 \mathrm{C}$ & 0.2120 & -0.1145 & 0.3873 & $0.013^{*}$ \\
$\mathrm{C} 2$ & $0.17844(17)$ & $-0.0169(5)$ & $0.5312(5)$ & $0.0126(9)$ \\
$\mathrm{C} 12$ & $0.15266(17)$ & $0.1803(5)$ & $0.5682(3)$ & $0.0141(7)$ \\
$\mathrm{H} 12 \mathrm{~A}$ & 0.1130 & 0.2060 & 0.5295 & $0.017^{*}$ \\
$\mathrm{H} 12 \mathrm{~B}$ & 0.1436 & 0.1749 & 0.6476 & $0.017^{*}$ \\
$\mathrm{O} 112$ & $0.19500(12)$ & $0.3372(3)$ & $0.5474(2)$ & $0.0174(6)$ \\
$\mathrm{H} 112$ & 0.2293 & 0.3138 & 0.5778 & $0.026^{*}$ \\
$\mathrm{C} 22$ & $0.23218(16)$ & $-0.0833(5)$ & $0.6035(3)$ & $0.0150(7)$ \\
$\mathrm{H} 22 \mathrm{~A}$ & 0.2164 & -0.1076 & 0.6784 & $0.018^{*}$ \\
$\mathrm{H} 22 \mathrm{~B}$ & 0.2489 & -0.2075 & 0.5748 & $0.018^{*}$ \\
$\mathrm{O} 212$ & $0.28111(11)$ & $0.0562(4)$ & $0.6084(2)$ & $0.0205(6)$ \\
$\mathrm{H} 212$ & 0.3083 & 0.0288 & 0.5614 & $0.031^{*}$ \\
$\mathrm{C} 32$ & $0.12571(16)$ & $-0.1669(5)$ & $0.5331(3)$ & $0.0139(7)$ \\
$\mathrm{H} 32 \mathrm{~A}$ & 0.1175 & -0.2067 & 0.6096 & $0.017^{*}$ \\
$\mathrm{H} 32 \mathrm{~B}$ & 0.0872 & -0.1067 & 0.5038 & $0.017^{*}$ \\
O312 & $0.14087(12)$ & $-0.3355(3)$ & $0.4693(2)$ & $0.0149(5)$ \\
$\mathrm{H} 312$ & 0.1612 & -0.4143 & 0.5080 & $0.022^{*}$ \\
& & & & \\
\hline
\end{tabular}

Atomic displacement parameters $\left(\AA^{2}\right)$

\begin{tabular}{lllllll}
\hline & $U^{11}$ & $U^{22}$ & $U^{33}$ & $U^{12}$ & $U^{13}$ & $U^{23}$ \\
\hline $\mathrm{Re} 1$ & $0.01274(6)$ & $0.01028(5)$ & $0.01470(9)$ & $-0.00034(4)$ & $0.00299(6)$ & $0.00074(6)$ \\
O11 & $0.0195(15)$ & $0.0232(14)$ & $0.0373(17)$ & $-0.0036(11)$ & $0.0120(13)$ & $-0.0001(13)$ \\
O21 & $0.0299(16)$ & $0.0150(12)$ & $0.0201(14)$ & $-0.0036(10)$ & $0.0061(12)$ & $0.0004(10)$ \\
O31 & $0.0252(15)$ & $0.0141(12)$ & $0.0218(14)$ & $0.0036(10)$ & $0.0011(12)$ & $-0.0006(10)$ \\
O41 & $0.0268(17)$ & $0.0171(14)$ & $0.0137(18)$ & $-0.0031(10)$ & $-0.0014(13)$ & $0.0013(11)$ \\
Re2 & $0.01365(7)$ & $0.01088(5)$ & $0.01303(8)$ & $-0.00034(5)$ & $0.00093(6)$ & $-0.00031(8)$ \\
O12 & $0.0235(16)$ & $0.0188(12)$ & $0.0180(18)$ & $-0.0037(11)$ & $-0.0066(12)$ & $-0.0068(13)$ \\
O22 & $0.0284(16)$ & $0.0237(14)$ & $0.0195(14)$ & $0.0086(11)$ & $0.0016(12)$ & $0.0010(11)$ \\
O32 & $0.0176(14)$ & $0.0194(13)$ & $0.0336(15)$ & $-0.0005(10)$ & $0.0063(11)$ & $0.0011(12)$ \\
O42 & $0.0393(17)$ & $0.0163(12)$ & $0.0210(15)$ & $-0.0084(11)$ & $0.0083(12)$ & $-0.0057(10)$ \\
N1 & $0.0087(13)$ & $0.0032(10)$ & $0.014(2)$ & $-0.0006(9)$ & $0.0076(14)$ & $0.0021(11)$ \\
C1 & $0.0109(18)$ & $0.0078(15)$ & $0.014(3)$ & $-0.0007(13)$ & $0.0004(14)$ & $0.0006(11)$ \\
C11 & $0.0146(19)$ & $0.0104(16)$ & $0.022(2)$ & $-0.0019(13)$ & $0.0032(15)$ & $-0.0018(14)$ \\
O111 & $0.0204(14)$ & $0.0121(12)$ & $0.0158(13)$ & $-0.0001(10)$ & $0.0032(11)$ & $-0.0008(9)$ \\
C21 & $0.0166(19)$ & $0.0093(16)$ & $0.022(2)$ & $-0.0026(13)$ & $0.0030(16)$ & $-0.0016(14)$ \\
O211 & $0.0208(15)$ & $0.0106(11)$ & $0.0256(16)$ & $-0.0020(10)$ & $-0.0012(12)$ & $0.0010(11)$ \\
C31 & $0.016(2)$ & $0.0167(19)$ & $0.018(2)$ & $0.0009(15)$ & $0.0001(15)$ & $0.0032(15)$ \\
O311 & $0.0127(14)$ & $0.0207(14)$ & $0.0250(16)$ & $-0.0001(11)$ & $-0.0028(12)$ & $-0.0077(12)$
\end{tabular}


supporting information

\begin{tabular}{lllllll}
$\mathrm{N} 2$ & $0.0144(13)$ & $0.0044(12)$ & $0.013(2)$ & $0.0016(9)$ & $-0.0018(13)$ & $-0.0049(10)$ \\
$\mathrm{C} 2$ & $0.019(2)$ & $0.0092(17)$ & $0.010(2)$ & $0.0024(12)$ & $0.0001(15)$ & $-0.0013(13)$ \\
$\mathrm{C} 12$ & $0.0154(19)$ & $0.0093(15)$ & $0.0176(19)$ & $0.0010(12)$ & $0.0031(14)$ & $-0.0003(13)$ \\
$\mathrm{O} 112$ & $0.0152(14)$ & $0.0083(11)$ & $0.0287(16)$ & $-0.0024(10)$ & $-0.0020(12)$ & $-0.0010(10)$ \\
$\mathrm{C} 22$ & $0.0139(19)$ & $0.0131(16)$ & $0.0182(19)$ & $0.0004(12)$ & $-0.0014(14)$ & $0.0007(14)$ \\
$\mathrm{O} 212$ & $0.0124(14)$ & $0.0215(13)$ & $0.0276(16)$ & $-0.0009(10)$ & $-0.0023(11)$ & $-0.0056(12)$ \\
$\mathrm{C} 32$ & $0.0139(19)$ & $0.0098(16)$ & $0.018(2)$ & $-0.0037(12)$ & $0.0023(15)$ & $-0.0022(13)$ \\
$\mathrm{O} 312$ & $0.0173(14)$ & $0.0093(11)$ & $0.0182(14)$ & $0.0009(9)$ & $-0.0011(11)$ & $-0.0013(9)$ \\
\hline
\end{tabular}

Geometric parameters $\left(\AA,{ }^{\circ}\right)$

\begin{tabular}{|c|c|c|c|}
\hline $\mathrm{Re} 1-\mathrm{O} 11$ & $1.736(2)$ & $\mathrm{C} 31-\mathrm{O} 311$ & $1.423(4)$ \\
\hline $\mathrm{Re} 1-\mathrm{O} 21$ & $1.728(2)$ & $\mathrm{C} 31-\mathrm{H} 31 \mathrm{~A}$ & 0.9900 \\
\hline $\mathrm{Re} 1-\mathrm{O} 31$ & $1.727(2)$ & $\mathrm{C} 31-\mathrm{H} 31 \mathrm{~B}$ & 0.9900 \\
\hline $\mathrm{Re} 1-\mathrm{O} 41$ & $1.702(5)$ & $\mathrm{O} 311-\mathrm{H} 311$ & 0.8400 \\
\hline $\mathrm{Re} 2-\mathrm{O} 12$ & $1.728(4)$ & $\mathrm{N} 2-\mathrm{C} 2$ & $1.519(7)$ \\
\hline $\mathrm{Re} 2-\mathrm{O} 22$ & $1.730(3)$ & $\mathrm{N} 2-\mathrm{H} 2 \mathrm{~A}$ & 0.9100 \\
\hline $\mathrm{Re} 2-\mathrm{O} 32$ & $1.736(3)$ & $\mathrm{N} 2-\mathrm{H} 2 \mathrm{~B}$ & 0.9100 \\
\hline $\mathrm{Re} 2-\mathrm{O} 42$ & $1.726(2)$ & $\mathrm{N} 2-\mathrm{H} 2 \mathrm{C}$ & 0.9100 \\
\hline $\mathrm{N} 1-\mathrm{C} 1$ & $1.520(7)$ & $\mathrm{C} 2-\mathrm{C} 22$ & $1.522(6)$ \\
\hline $\mathrm{N} 1-\mathrm{H} 1 \mathrm{~A}$ & 0.9100 & $\mathrm{C} 2-\mathrm{C} 32$ & $1.530(5)$ \\
\hline N1-H1B & 0.9100 & $\mathrm{C} 2-\mathrm{C} 12$ & $1.531(5)$ \\
\hline $\mathrm{N} 1-\mathrm{H} 1 \mathrm{C}$ & 0.9100 & $\mathrm{C} 12-\mathrm{O} 112$ & $1.432(4)$ \\
\hline $\mathrm{C} 1-\mathrm{C} 31$ & $1.528(6)$ & $\mathrm{C} 12-\mathrm{H} 12 \mathrm{~A}$ & 0.9900 \\
\hline $\mathrm{C} 1-\mathrm{C} 21$ & $1.529(5)$ & $\mathrm{C} 12-\mathrm{H} 12 \mathrm{~B}$ & 0.9900 \\
\hline $\mathrm{C} 1-\mathrm{C} 11$ & $1.537(4)$ & $\mathrm{O} 112-\mathrm{H} 112$ & 0.8400 \\
\hline $\mathrm{C} 11-\mathrm{O} 111$ & $1.427(4)$ & $\mathrm{C} 22-\mathrm{O} 212$ & $1.422(4)$ \\
\hline C11-H11A & 0.9900 & $\mathrm{C} 22-\mathrm{H} 22 \mathrm{~A}$ & 0.9900 \\
\hline C11-H11B & 0.9900 & $\mathrm{C} 22-\mathrm{H} 22 \mathrm{~B}$ & 0.9900 \\
\hline $\mathrm{O} 111-\mathrm{H} 111$ & 0.8400 & $\mathrm{O} 212-\mathrm{H} 212$ & 0.8400 \\
\hline $\mathrm{C} 21-\mathrm{O} 211$ & $1.431(4)$ & $\mathrm{C} 32-\mathrm{O} 312$ & $1.433(4)$ \\
\hline $\mathrm{C} 21-\mathrm{H} 21 \mathrm{~A}$ & 0.9900 & $\mathrm{C} 32-\mathrm{H} 32 \mathrm{~A}$ & 0.9900 \\
\hline $\mathrm{C} 21-\mathrm{H} 21 \mathrm{~B}$ & 0.9900 & C32- H32B & 0.9900 \\
\hline $\mathrm{O} 211-\mathrm{H} 211$ & 0.8400 & $\mathrm{O} 312-\mathrm{H} 312$ & 0.8400 \\
\hline $\mathrm{O} 41-\mathrm{Re} 1-\mathrm{O} 31$ & $109.6(2)$ & $\mathrm{O} 311-\mathrm{C} 31-\mathrm{H} 31 \mathrm{~A}$ & 109.2 \\
\hline $\mathrm{O} 41-\mathrm{Re} 1-\mathrm{O} 21$ & $108.7(2)$ & $\mathrm{C} 1-\mathrm{C} 31-\mathrm{H} 31 \mathrm{~A}$ & 109.2 \\
\hline $\mathrm{O} 31-\mathrm{Re} 1-\mathrm{O} 21$ & $108.7(2)$ & $\mathrm{O} 311-\mathrm{C} 31-\mathrm{H} 31 \mathrm{~B}$ & 109.2 \\
\hline $\mathrm{O} 41-\mathrm{Re} 1-\mathrm{O} 11$ & $110.6(2)$ & $\mathrm{C} 1-\mathrm{C} 31-\mathrm{H} 31 \mathrm{~B}$ & 109.2 \\
\hline $\mathrm{O} 31-\mathrm{Re} 1-\mathrm{O} 11$ & $110.0(2)$ & $\mathrm{H} 31 \mathrm{~A}-\mathrm{C} 31-\mathrm{H} 31 \mathrm{~B}$ & 107.9 \\
\hline $\mathrm{O} 21-\mathrm{Re} 1-\mathrm{O} 11$ & $109.2(2)$ & $\mathrm{C} 31-\mathrm{O} 311-\mathrm{H} 311$ & 109.5 \\
\hline $\mathrm{O} 42-\mathrm{Re} 2-\mathrm{O} 12$ & $108.4(2)$ & $\mathrm{C} 2-\mathrm{N} 2-\mathrm{H} 2 \mathrm{~A}$ & 109.5 \\
\hline $\mathrm{O} 42-\mathrm{Re} 2-\mathrm{O} 22$ & $109.4(2)$ & $\mathrm{C} 2-\mathrm{N} 2-\mathrm{H} 2 \mathrm{~B}$ & 109.5 \\
\hline $\mathrm{O} 12-\mathrm{Re} 2-\mathrm{O} 22$ & $109.6(2)$ & $\mathrm{H} 2 \mathrm{~A}-\mathrm{N} 2-\mathrm{H} 2 \mathrm{~B}$ & 109.5 \\
\hline $\mathrm{O} 42-\mathrm{Re} 2-\mathrm{O} 32$ & $109.3(2)$ & $\mathrm{C} 2-\mathrm{N} 2-\mathrm{H} 2 \mathrm{C}$ & 109.5 \\
\hline $\mathrm{O} 12-\mathrm{Re} 2-\mathrm{O} 32$ & $110.8(2)$ & $\mathrm{H} 2 \mathrm{~A}-\mathrm{N} 2-\mathrm{H} 2 \mathrm{C}$ & 109.5 \\
\hline $\mathrm{O} 22-\mathrm{Re} 2-\mathrm{O} 32$ & $109.3(2)$ & $\mathrm{H} 2 \mathrm{~B}-\mathrm{N} 2-\mathrm{H} 2 \mathrm{C}$ & 109.5 \\
\hline $\mathrm{C} 1-\mathrm{N} 1-\mathrm{H} 1 \mathrm{~A}$ & 109.5 & $\mathrm{~N} 2-\mathrm{C} 2-\mathrm{C} 22$ & $110.5(3)$ \\
\hline
\end{tabular}




\begin{tabular}{|c|c|c|c|}
\hline $\mathrm{C} 1-\mathrm{N} 1-\mathrm{H} 1 \mathrm{~B}$ & 109.5 & $\mathrm{~N} 2-\mathrm{C} 2-\mathrm{C} 32$ & $107.5(4)$ \\
\hline $\mathrm{H} 1 \mathrm{~A}-\mathrm{N} 1-\mathrm{H} 1 \mathrm{~B}$ & 109.5 & $\mathrm{C} 22-\mathrm{C} 2-\mathrm{C} 32$ & $110.5(3)$ \\
\hline $\mathrm{C} 1-\mathrm{N} 1-\mathrm{H} 1 \mathrm{C}$ & 109.5 & $\mathrm{~N} 2-\mathrm{C} 2-\mathrm{C} 12$ & $107.8(3)$ \\
\hline $\mathrm{H} 1 \mathrm{~A}-\mathrm{N} 1-\mathrm{H} 1 \mathrm{C}$ & 109.5 & $\mathrm{C} 22-\mathrm{C} 2-\mathrm{C} 12$ & $111.5(4)$ \\
\hline $\mathrm{H} 1 \mathrm{~B}-\mathrm{N} 1-\mathrm{H} 1 \mathrm{C}$ & 109.5 & $\mathrm{C} 32-\mathrm{C} 2-\mathrm{C} 12$ & $108.9(3)$ \\
\hline $\mathrm{N} 1-\mathrm{C} 1-\mathrm{C} 31$ & $109.3(3)$ & $\mathrm{O} 112-\mathrm{C} 12-\mathrm{C} 2$ & $112.6(3)$ \\
\hline $\mathrm{N} 1-\mathrm{C} 1-\mathrm{C} 21$ & $108.4(3)$ & $\mathrm{O} 112-\mathrm{C} 12-\mathrm{H} 12 \mathrm{~A}$ & 109.1 \\
\hline $\mathrm{C} 31-\mathrm{C} 1-\mathrm{C} 21$ & $111.4(4)$ & $\mathrm{C} 2-\mathrm{C} 12-\mathrm{H} 12 \mathrm{~A}$ & 109.1 \\
\hline $\mathrm{N} 1-\mathrm{C} 1-\mathrm{C} 11$ & $107.5(4)$ & $\mathrm{O} 112-\mathrm{C} 12-\mathrm{H} 12 \mathrm{~B}$ & 109.1 \\
\hline $\mathrm{C} 31-\mathrm{C} 1-\mathrm{C} 11$ & $110.9(3)$ & $\mathrm{C} 2-\mathrm{C} 12-\mathrm{H} 12 \mathrm{~B}$ & 109.1 \\
\hline $\mathrm{C} 21-\mathrm{C} 1-\mathrm{C} 11$ & $109.2(3)$ & $\mathrm{H} 12 \mathrm{~A}-\mathrm{C} 12-\mathrm{H} 12 \mathrm{~B}$ & 107.8 \\
\hline $\mathrm{O} 111-\mathrm{C} 11-\mathrm{C} 1$ & $111.9(3)$ & $\mathrm{C} 12-\mathrm{O} 112-\mathrm{H} 112$ & 109.5 \\
\hline $\mathrm{O} 111-\mathrm{C} 11-\mathrm{H} 11 \mathrm{~A}$ & 109.2 & $\mathrm{O} 212-\mathrm{C} 22-\mathrm{C} 2$ & $112.4(3)$ \\
\hline $\mathrm{C} 1-\mathrm{C} 11-\mathrm{H} 11 \mathrm{~A}$ & 109.2 & $\mathrm{O} 212-\mathrm{C} 22-\mathrm{H} 22 \mathrm{~A}$ & 109.1 \\
\hline $\mathrm{O} 111-\mathrm{C} 11-\mathrm{H} 11 \mathrm{~B}$ & 109.2 & $\mathrm{C} 2-\mathrm{C} 22-\mathrm{H} 22 \mathrm{~A}$ & 109.1 \\
\hline $\mathrm{C} 1-\mathrm{C} 11-\mathrm{H} 11 \mathrm{~B}$ & 109.2 & $\mathrm{O} 212-\mathrm{C} 22-\mathrm{H} 22 \mathrm{~B}$ & 109.1 \\
\hline $\mathrm{H} 11 \mathrm{~A}-\mathrm{C} 11-\mathrm{H} 11 \mathrm{~B}$ & 107.9 & $\mathrm{C} 2-\mathrm{C} 22-\mathrm{H} 22 \mathrm{~B}$ & 109.1 \\
\hline C11-O111-H111 & 109.5 & $\mathrm{H} 22 \mathrm{~A}-\mathrm{C} 22-\mathrm{H} 22 \mathrm{~B}$ & 107.8 \\
\hline $\mathrm{O} 211-\mathrm{C} 21-\mathrm{C} 1$ & $113.3(3)$ & $\mathrm{C} 22-\mathrm{O} 212-\mathrm{H} 212$ & 109.5 \\
\hline $\mathrm{O} 211-\mathrm{C} 21-\mathrm{H} 21 \mathrm{~A}$ & 108.9 & $\mathrm{O} 312-\mathrm{C} 32-\mathrm{C} 2$ & $111.6(3)$ \\
\hline $\mathrm{C} 1-\mathrm{C} 21-\mathrm{H} 21 \mathrm{~A}$ & 108.9 & $\mathrm{O} 312-\mathrm{C} 32-\mathrm{H} 32 \mathrm{~A}$ & 109.3 \\
\hline $\mathrm{O} 211-\mathrm{C} 21-\mathrm{H} 21 \mathrm{~B}$ & 108.9 & $\mathrm{C} 2-\mathrm{C} 32-\mathrm{H} 32 \mathrm{~A}$ & 109.3 \\
\hline $\mathrm{C} 1-\mathrm{C} 21-\mathrm{H} 21 \mathrm{~B}$ & 108.9 & $\mathrm{O} 312-\mathrm{C} 32-\mathrm{H} 32 \mathrm{~B}$ & 109.3 \\
\hline $\mathrm{H} 21 \mathrm{~A}-\mathrm{C} 21-\mathrm{H} 21 \mathrm{~B}$ & 107.7 & $\mathrm{C} 2-\mathrm{C} 32-\mathrm{H} 32 \mathrm{~B}$ & 109.3 \\
\hline $\mathrm{C} 21-\mathrm{O} 211-\mathrm{H} 211$ & 109.5 & $\mathrm{H} 32 \mathrm{~A}-\mathrm{C} 32-\mathrm{H} 32 \mathrm{~B}$ & 108.0 \\
\hline $\mathrm{O} 311-\mathrm{C} 31-\mathrm{C} 1$ & $112.0(3)$ & $\mathrm{C} 32-\mathrm{O} 312-\mathrm{H} 312$ & 109.5 \\
\hline $\mathrm{N} 1-\mathrm{C} 1-\mathrm{C} 11-\mathrm{O} 111$ & $-47.2(4)$ & $\mathrm{N} 2-\mathrm{C} 2-\mathrm{C} 12-\mathrm{O} 112$ & $-51.4(4)$ \\
\hline $\mathrm{N} 1-\mathrm{C} 1-\mathrm{C} 21-\mathrm{O} 211$ & $51.0(4)$ & $\mathrm{N} 2-\mathrm{C} 2-\mathrm{C} 22-\mathrm{O} 212$ & $64.0(4)$ \\
\hline $\mathrm{N} 1-\mathrm{C} 1-\mathrm{C} 31-\mathrm{O} 311$ & $-63.1(4)$ & $\mathrm{N} 2-\mathrm{C} 2-\mathrm{C} 32-\mathrm{O} 312$ & 45.7 (4) \\
\hline
\end{tabular}

Hydrogen-bond geometry $\left(A,{ }^{\circ}\right)$

\begin{tabular}{lllll}
\hline$D-\mathrm{H} \cdots A$ & $D-\mathrm{H}$ & $\mathrm{H} \cdots A$ & $D \cdots A$ & $D-\mathrm{H}^{\cdots} A$ \\
\hline $\mathrm{N} 2-\mathrm{H} 2 A \cdots \mathrm{O} 21$ & 0.91 & 2.03 & $2.858(4)$ & 150 \\
$\mathrm{~N} 2-\mathrm{H} 2 B \cdots \mathrm{O} 111^{\mathrm{i}}$ & 0.91 & 1.88 & $2.788(4)$ & 173 \\
$\mathrm{~N} 2-\mathrm{H} 2 C \cdots \mathrm{O} 31^{\mathrm{ii}}$ & 0.91 & 1.98 & $2.879(4)$ & 169 \\
$\mathrm{O} 112-\mathrm{H} 112 \cdots \mathrm{O} 212$ & 0.84 & 2.12 & $2.773(4)$ & 134 \\
$\mathrm{O} 112-\mathrm{H} 112 \cdots \mathrm{O} 41$ & 0.84 & 2.49 & $2.942(4)$ & 115 \\
$\mathrm{O} 212-\mathrm{H} 212 \cdots \mathrm{O} 32^{\mathrm{ii}}$ & 0.84 & 1.89 & $2.721(4)$ & 168 \\
$\mathrm{O} 312-\mathrm{H} 312 \cdots \mathrm{O} 112^{\mathrm{ii}}$ & 0.84 & 1.92 & $2.704(4)$ & 156 \\
$\mathrm{~N} 1-\mathrm{H} 1 A \cdots \mathrm{O} 312^{\mathrm{iii}}$ & 0.91 & 1.83 & $2.738(4)$ & 176 \\
$\mathrm{~N} 1-\mathrm{H} 1 B \cdots \mathrm{O} 42^{\mathrm{ii}}$ & 0.91 & 2.05 & $2.872(4)$ & 150 \\
$\mathrm{~N} 1-\mathrm{H} 1 C \cdots \mathrm{O} 22$ & 0.91 & 1.98 & $2.862(4)$ & 164 \\
$\mathrm{O} 111-\mathrm{H} 111 \cdots \mathrm{O} 211^{\mathrm{ii}}$ & 0.84 & 1.89 & $2.681(3)$ & 157 \\
$\mathrm{O} 211-\mathrm{H} 211 \cdots \mathrm{O} 311$ & 0.84 & 2.13 & $2.774(4)$ & 134
\end{tabular}


supporting information

$\mathrm{O} 211-\mathrm{H} 211 \cdots \mathrm{O} 12^{\mathrm{ii}}$

$\mathrm{O} 311-\mathrm{H} 311 \cdots \mathrm{O} 11$

0.84

2.54

$2.960(4)$

$2.714(4)$

112

1.88

170

Symmetry codes: (i) $x-1 / 2,-y+1, z$; (ii) $x, y-1, z$; (iii) $x+1 / 2,-y, z$; (iv) $x, y+1, z$. 